\title{
Methods for Geometric Modeling of Electronic Inspection Data
}

\author{
J. Rodney Taylor \\ Computer Aided Engineering \\ Lockheed Martin Energy Systems, Inc.
}

June 28, 1999

Approved for Public Release;

distribution is unlimited

Prepared by the

Oak Ridge Y-12 Plant

Managed by

Lockheed Martin Energy Systems, Inc.

for the

U.S. Department of Energy

Under contract DE-AC05-84OR21400 


\section{DISCLAIMER}

This report was prepared as an account of work sponsored by an agency of the United States Government. Neither the United States Government nor any agency thereof, nor any of their employees, makes any warranty, express or implied, or assumes any legal liability or responsibility for the accuracy, completeness, or usefulness of any information, apparatus, product, or process disclosed, or represents that its use would not infringe privately owned rights. Reference herein to any specific commercial product, process, or service by trade name, trademark, manufacturer, or otherwise, does not necessarily constitute or imply its endorsement, recommendation, or favoring by the United States Government or any agency thereof. The views and opinions of authors expressed herein do not necessarily state or reflect those of the United States Government or any agency thereof. 


\begin{abstract}
The Oak Ridge Y-12 plant has implemented a methodology for characterizing parts and assemblies based on their as-measured condition. Complete solid models of each part in an assembly are created to represent these measurements. The models produced can be analyzed for mass properties, relative shape variations, interference checking, and optimal mating between components. This report describes the methodology used to take a relatively sparse set of measured inspection points and generate a representative fully analytical solid model.
\end{abstract}

\title{
Introduction
}

YZ, Dual YZ, and Coordinate Measuring Machines (CMMs) are precision inspection machines used to obtain dimensional information about manufactured and assembled parts. YZ and Dual YZ machines are used to obtain inner and outer contour information. CMMs are used for feature measurements. Current dimensional inspection technologies provide for the capture and archival of surface contour and feature information. At the Y-12 plant, modern controllers have been installed on machines that measure the deviation along the surface normal relative to a point on the nominal part. This information is primarily used to assure that the manufactured part is within the tolerance band prescribed. Advanced modeling techniques have been developed which automatically apply the measured point onto the nominal shape to create an as-measured model. These as-measured models can then be incorporated into the nominal assembly coordinate system to generate an assembled unit.

\section{Consolidated Measurement Files}

These inspection machines generate ASCII text history files that serve as records of each part's surface contour and feature information. While these files are useful for archival purposes, they are generally difficult for most people to read and understand. What is needed is a file format that incorporates the data contained in the history files but in an organized and easierto-understand format.

At Y-12 this file is referred to as a consolidated measurement file and is derived from information in the YZ and Dual YZ history files. The measurement file contains header information that includes the name of the history file from which the measurement file was generated, the drawing number of the part that was inspected, the inspection machine, and the maximum and minimum measurements for each contour. Data in the file is organized into longitudinal sweeps; each sweep being comprised of points lying on the various contours of the part.

Each inspected part has its own part coordinate system. This coordinate system is located at the intersection of the primary datums and is oriented such that the positive $\mathrm{Z}$ axis passes through the body of the part and the positive $\mathrm{X}$ axis passes through an identifying mark on the part outer surface. All points in the measurement file are relative to this coordinate system. 
For each inspected point on the part the measurement file records in Cartesian coordinates the measured point, aligned point, nominal point, and surface normal. Additionally the measurement file records contour deviation and wall thickness deviation. The measured point is the $(\mathrm{x}, \mathrm{y}, \mathrm{z})$ point that was actually touched by the inspection probe. The aligned point is the $(\mathrm{x}, \mathrm{y}, \mathrm{z})$ point that the probe would have touched had it performed the measurement exactly at the azimuth angle called for in the inspection program. Nominal point is the $(\mathrm{x}, \mathrm{y}, \mathrm{z})$ point that the inspection program was attempting to measure. Surface normal is the $(\mathrm{x}, \mathrm{y}, \mathrm{z})$ unit vector that represents the normal vector at the nominal point on the surface. The contour deviation is a scalar value that represents the amount by which the inspected point deviated from the nominal surface on the part. In all cases a positive value for contour deviation indicates excess stock material; a negative value indicates a lack of material. Wall thickness is a scalar value that represents the distance between measured points on the inner and outer contour of the object.

In addition to the measurement file an assembly contour file is generated for storing inspection data. This file records the same information contained in the measurement file but in a different format. While the measurement file stores inspection data in Cartesian coordinates, the assembly contour file stores the data in spherical coordinates. Additionally, while the measurement file records inspection points in part specific coordinates, the assembly contour file records inspection points as if the part were in its nominal location in assembly coordinates.

Measurement files are stored using the .mea extension. Assembly contour files are stored using the .acc extension.

\section{Consolidated Feature Files}

CMMs are used to measure the part features not measured by the YZ and Dual YZ machines. Generally these are points located in form features such as slots, holes and keyways. As with $\mathrm{YZ}$ and Dual YZ machines, data from the CMMs are recorded in history files that are generally difficult for most people to read and understand. The information in the history file is therefore translated into an organized and easier-to-understand format.

At Y-12 this file is referred to as the feature file and is derived from information in the CMM history file. The feature file contains header information that includes the name of the history file from which the feature file was generated, the drawing number of the part that was inspected, the inspection machine, and the maximum and minimum measurements for each contour. Data in the file is organized by feature name; each feature being comprised of points lying on the various surfaces of the part. Each inspected part has a part coordinate system. This coordinate system is located at the intersection of the primary datums and is oriented such that the positive $\mathrm{Z}$ axis passes through the body of the part and the positive $\mathrm{X}$ axis passes through an identifying mark on the part outer surface. All points in the feature file are relative to this coordinate system.

For each inspected point on the part the measurement file records in Cartesian coordinates the measured point and surface normal. Additionally, the measurement file records the contour deviation. The measured point is the $(\mathrm{x}, \mathrm{y}, \mathrm{z})$ point that was actually touched by the inspection 
probe. Surface normal is the $(\mathrm{x}, \mathrm{y}, \mathrm{z})$ unit vector that represents the normal vector at the nominal point. The contour deviation is a scalar value that represents the amount by which the inspected point deviated from the nominal surface point on the part. In all cases a positive value for contour deviation indicates excess stock material; a negative value indicates a lack of material.

In addition to the feature file an assembly feature file is generated for storing inspection data. This file records the same information contained in the feature file but in a different format. While the feature file stores inspection data in Cartesian coordinates, the assembly feature file stores the data in spherical coordinates. Additionally, while the feature file records inspection points in part specific coordinates, the assembly feature file records inspection points as if the part were in its nominal location in assembly coordinates.

Feature files are stored using the .fea extension. Assembly feature files are stored using the .acf extension.

\section{As-Built Solid Model Creation}

This measurement file contains inspection data for each measured contour on the piece part. We desire to create a solid model that accurately represents each point contained in the measurement file. We use the $\mathrm{CAD}$ system Pro/Engineer (Pro/E) for this purpose. Pro/E offers several features that make it suitable for this task. Firstly, it permits the user to create solid models of piece parts. These models are analytical and therefore can be used to investigate mass properties. Secondly, Pro/E has an application programming interface language built into it that lets the user extend base Pro/E functionality. Thirdly, Pro/E is widely used across the nuclear weapons complex. This permits models and programs created at one site to be shared with other labs in the NWC.

The first step in creating the solid model is to generate a rotational blend solid template in Pro/E. This template does not have to look exactly like the nominal piece part but must share certain characteristics with the nominal model. These are end point tangency constraints, nominal edge point constraints, and equal number of longitudinal inspection points. Creation of the solid template is a manual process and is quite tedious and time-consuming. Just as the actual part is inspected every four degrees of longitude, the template contains point sets every four degrees as well. These point sets are planar and lie along the various contours in the template. For each contour represented in the longitudinal point set, the modeler must create a spline that passes through the points and exhibits the desired end conditions. These splines form a closed loop and lie in the same plane as the point set from which they are generated. In addition to the closed loop splines the modeler must create an axis of revolution that is used to revolve about while creating the solid. Sweeping each closed spline loop about the axis of revolution generates the rotational blend solid. It is this solid that serves as the template for subsequent operations.

Once the solid exists in Pro/E it saved in a template directory and used by the modeler to create each instance of the as-built solid model. Using a Pro/Toolkit application the modeler is able to specify in Pro/E which measurement file to apply to the template model. Pro/E will then 
perturb the template so that each aligned point in the measurement file now lies on the surface of the solid template. Each instance of this as-built piece part model is then archived for future analysis.

\section{Ray Trace Analysis}

The Pro/E user may perform a ray trace analysis of the as-built part. Ray trace analysis provides the ability to interrogate the as-built model and determine the location of various points on the surface of the model. The interrogated points may or may not be the same points that were used to create the as-built model in the first place. Generally, ray tracing involves projecting rays in a spherical pattern from a given coordinate system (usually located at the part center) and determining where the rays pierce the various surfaces of the model. The user may specify a range of angles both in the elevation and azimuth direction. Elevation angle may range from north pole to south pole or 0 to 180 degrees respectively. Azimuth angle may range from 0 to 360 degrees about the equator. Additionally the user may specify the angular step size to use while projecting the various rays into the model. Once the user has performed a ray trace analysis of a particular model he can then store the results in an ASCII text file. The file will summarize the findings of the ray trace analysis.

\section{VRML Models}

The measurement file is used to create a VRML model of the inspected part. This VRML model may be viewed in a web browser and is useful for displaying dimensional inspection data in a visual, easy to understand format. The VRML model is presented to the client workstation in two frames. The left-hand frame contains the 3D solid model of the inspected part. This model is rendered to scale and is colored coded. The colors at each point on the model represent the deviation of that point from its nominal value. For both the inner and outer contour of the part, the VRML model may display either the contour deviation or the wall thickness deviation. The right-hand frame contains administrative information about the model. This information includes a color legend specifying the upper and lower tolerance, part drawing number, part serial number, and "wall thickness/contour" deviation specifier for the inner and outer contours of the part.

\section{As-Built Assembly Model Creation}

Following the aforementioned technique for creating as-built solid models, each component in the assembly is modeled in Pro/E to its as-built condition. Using Pro/E's assembly creation context the user brings each as-built component into the assembly placing it into its nominal location based on scribe lines in the model. After the assembly is generated the user stores the model for future analysis. With this as-built assembly model the user can perform ray trace analysis as with the part model. In the Pro/E assembly context, however, rays traces are projected through the entire assembly and intersection points are recorded for each part comprising it. This capability makes it possible to look for interferences between mating parts in the assembly. As with ray trace analysis in the Pro/E part context, the user may store the results in an ASCII text file. An additional capability in Pro/E when checking for interferences 
is volumetric analysis. This capability allows the user to display on screen any volumes of overlap between mating parts in the assembly. The amount of overlapping volume is computed and displayed to the user as well. The user is then able to determine whether the components in the as-built assembly will properly join to each other.

\section{Summary}

The methodology described above was used to create Pro/Engineer as-measured solid models and assemblies for demonstration to personnel in the NWC. These models were useful for analyzing components in a current program at $\mathrm{Y}-12$. 


\section{$\underline{\text { Distribution }}$}

F. W. Jones, 9202, MS-8097

Y-12 Plant Records Services (3), 9711-5, MS-8169 